\title{
Numerical simulation of leaking hydrogen dispersion behavior in a partially open space
}

\author{
K. Matsuura ${ }^{1}$, H. Kanayama ${ }^{1}$, H. Tsukikawa ${ }^{2}$ \& M. Inoue ${ }^{3}$ \\ ${ }^{I}$ Department of Intelligent Machinery and Systems, Kyushu University, \\ Japan \\ ${ }^{2}$ Saibu Gas Co., Ltd., Japan \\ ${ }^{3}$ Department of Earth Resources Engineering, Kyushu University, Japan
}

\begin{abstract}
The numerical simulation of hydrogen dispersion in a partially open space is performed in this paper. The transient behavior of hydrogen and the process of accumulation in the space are discussed. Also, the effects of changing vent positions, vent conditions and surrounding atmospheric currents on the distribution of hydrogen concentrations are shown. Based on the results, conditions for preventing the dispersion and accumulation of hydrogen in the space, and the importance of incorporating a relationship with the outside environment in the computation, are discussed.
\end{abstract}

Keywords: dispersion, computational fluid dynamics, hazard modelling, ventilation, atmospheric current.

\section{Introduction}

Safety is recognized as one of the most important issues in promoting hydrogen utility. Accompanying its extensive usage, the possibility of accidental release is considered to increase in hydrogen infrastructure including storage, bulk transportation and distribution, production and utilization.

Various safety issues from a priori to a posterior have been discussed so far, such as hydrogen embrittlement, permeation, convective leakage, ignition and explosion. In particular, research on the leaking hydrogen is important to prevent accidental ignition, and is also considered to give the safety margin for leakage. Therefore, for the safe usage of hydrogen it is necessary to predict and understand its characteristics of leakage and dispersion. 
Regarding hydrogen dispersion, there have been reports on the evaluation of leak flow rate [1], the dispersion behaviors in urban [2] and residential areas $[3,4]$, and the design of ventilation systems [5], etc. In a series of researches, dispersion in a highly confined area is recognized as one of the most dangerous scenarios. In the literature, Swain and Swain [5] report the influence of building geometry and passive ventilation on the formation of combustible gas clouds. In [6], the authors discuss a method for hydrogen leak classification and propose risk assessment determination in partially open spaces.

However, there are few reports on the transient behavior in the process of accumulation, or the effects of ventilation on hydrogen concentration. Also, further investigation is thought to be necessary regarding structures that prevent dispersion and accumulation.

Using real hydrogen for the investigation requires a well-prepared facility to ensure the safety of the experiments. CFD is a cost-effective and safe approach, and if constitutive factors such as the chemical and physical model, turbulence model, boundary conditions, etc., are modeled properly, and numerical schemes are verified, the methodology gives much information on the integral linkage that increases the potential hazard. This information is also important for computation to prevent hazards.

Therefore in this paper, considering important factors that affect the dispersion behavior, namely vent position and vent pressure, hydrogen dispersion in a partially open space is investigated computationally [8]. The transient behavior of hydrogen and the process of accumulation in the space are discussed. Also, the effects of changing vent positions, vent conditions and the surrounding atmospheric currents on the distribution of hydrogen concentration are shown. Based on the results, conditions for preventing dispersion and accumulation of hydrogen in the space, and the importance of incorporating a relationship with the outside environment in computation, are discussed.

\section{Computational geometry}

Two computational domains are used in this study. One is a partially open space, a hallway, with the dimension of $L_{\mathrm{x}}=2.9[\mathrm{~m}], L_{\mathrm{y}}=1.22[\mathrm{~m}]$ and $L_{\mathrm{z}}=0.74[\mathrm{~m}]$ in x, $\mathrm{y}$ and $\mathrm{z}$ directions, respectively. Figure 1 shows the schematic of the hallway. Regarding computational conditions, pure hydrogen leaks into the space with a flow rate of 2 standard cubic feet per minute $\left(9.44 \times 10^{-4} \mathrm{~m}^{3} / \mathrm{s}\right)$. The area of the inflow orifice, the roof vent and the door vent are all $4.65 \times 10^{-2}\left[\mathrm{~m}^{2}\right]$.

In the computational domain, the time series data of hydrogen concentration are sampled at four positions shown in the left of fig. 1, and the data are compared with the corresponding experimental data. The right of fig. 1 shows the experimental model of the hallway, which is made of acrylic acid resin. At both the roof vent and the door vent, the condition of natural ventilation is imposed. The structure grid of H-type topology is generated in the hallway. The total number of grid points used finally is about $2.9 \times 10^{4}$ pts. The results of this domain are discussed in Section 4.1. 


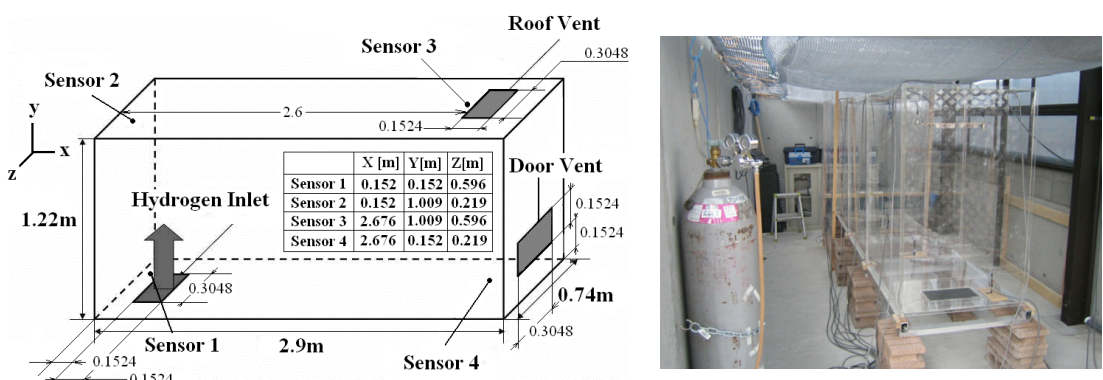

Figure 1: The hallway (left: schematic, right: experimental model).

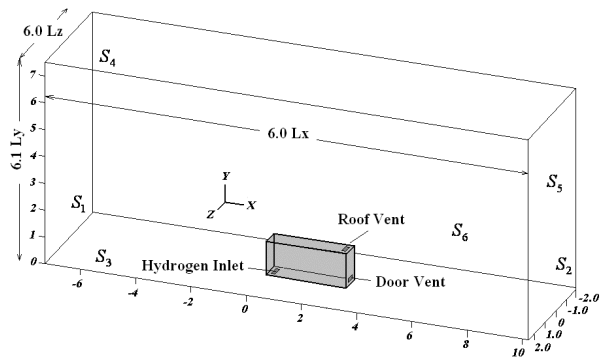

Figure 2: $\quad$ The schematic of the hallway model with outside space.

The other computational domain is a hallway with outside space. Figure 2 shows the schematic of it. This model is introduced to incorporate the effect of atmospheric currents outside the hallway on the behavior of hydrogen dispersion and accumulation. The computational domain has the dimensions of $6.0 L_{\mathrm{x}} \times 6.1$ $L_{\mathrm{y}} \times 6.0 L_{\mathrm{z}}$, and the hallway is placed on the center of the $\mathrm{y}=0$ plane. Only through the door vent and the roof vent the space inside the hallway is connected to the outside. The whole domain is divided into 20 blocks and the structure grid of H-type topology is generated in each block. The total number of grid points used finally is about $1.5 \times 10^{5}$ points. The results of this domain are discussed in Section 4.2.

\section{Numerical methods}

The governing equations are the continuity equation, the compressible Navier-Stokes equation with gravitational force, the energy equation and the transport equations for the mass fraction of $\mathrm{H}_{2}, \mathrm{~N}_{2}, \mathrm{O}_{2}$. Turbulence is modeled by the standard $k-\varepsilon$ model. Diffusion velocities in multicomponent diffusion are estimated by the Stenfan-Maxwell equation [8].

The above equations are solved by SIMPLEC. The accuracy of discretization is $2^{\text {nd }}$-order. Computations based on the above equations are performed by the commercial CFD software CFD-ACE+ Ver. 2006. Regarding boundary 
conditions for the single hallway, velocities and temperatures are specified at the inlet and a constant pressure is imposed at both the roof vent and the door vent. To take the change in static pressure, changes along the height due to the hydrostatic pressure are taken into consideration at the door vent. A duct is attached to the door vent, and the height of its exit boundary is set to be equal between the roof vent and the door vent. The effectiveness and the soundness of the treatment are confirmed by the comparison of the present results with those obtained for the hallway surrounded by the calm atmosphere. At the wall, a non-slip, adiabatic condition is imposed. Regarding chemical species, pure hydrogen is specified at the inlet orifice, extrapolation is used at the roof vent and air is specified at the door vent. Regarding turbulence quantities, i.e., $\mathrm{k}$ and $\varepsilon$, the turbulence intensity $\left(T u_{i n}\right)$ and the length scale $(l)$ are specified at the inlet and the two vents. Here, the turbulent energy at the inlet is related to the vertical velocity $\left(\mathrm{V}_{\text {in }}\right)$ and the turbulent intensity as $\mathrm{k}=3 / 2\left(T u_{\text {in }} \times V_{\text {in }}\right)^{2}$, and the dissipation rate is related to the turbulent energy and the length scale as $\varepsilon=C_{\mu} k^{3 / 2} / l$. Here, $T u_{\text {in }}, l$ and $C_{\mu}$ are taken to be 0.01, 0.3048 and 0.09, respectively. The time step width for unsteady computations is set to be 1 second. Regarding the hallway with outside space, boundary conditions for the outside space are varied depending on the direction and the velocity magnitude of the atmospheric current mentioned later in Section 4.2. Fixed velocities are imposed at both the inlet and the outlet, i.e., $S_{1}$ and $S_{2}$ when the direction of the atmospheric current is $\pm \mathrm{x}$, and symmetry is imposed at the lateral and the upper boundaries, i.e., $\mathrm{S}_{4}, \mathrm{~S}_{5}$ and $\mathrm{S}_{6}$ when the direction of the current is $\pm \mathrm{x}$. In all cases, a non-slip, adiabatic wall condition is imposed at $\mathrm{S}_{3}$. In this model, hydrostatic pressure is subtracted from the static pressure to impose the above conditions.

\section{Results and discussion}

\subsection{The effect of vent position and condition}

In fig. 3, the time histories of the computed hydrogen concentrations are compared with the experimental data. In the figure, two experimental data are shown. One is the data shown in the literature [6], and the other is the data measured by one of the authors, Inoue, using thermal conductivity sensors, which are appropriate for measuring the range of concentration in this study. Although the current sensor has a large time lag (around 10 seconds) due to its inherent time coefficient, it is one of the effective tools of measurement at present.

Regarding the data [6], the detail except for the experiment model and the condition for hydrogen inlet are not described. Two experimental data are shown for their consistency. Although relatively large differences are observed between the present results and the experimental data at Sensor 1, and the data at Sensor 4 , the present results agree well with the experimental data at Sensor 2 and Sensor 3, and those after 150 seconds at Sensor 1. 


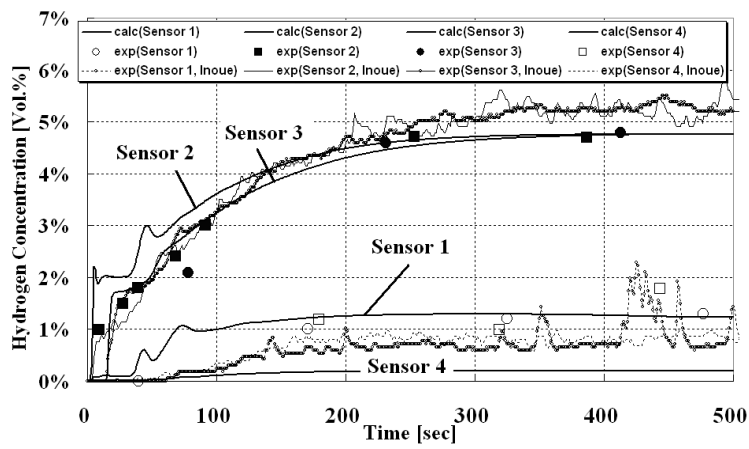

Figure 3: Comparisons of the time histories of hydrogen concentration between the computations and the experiments.

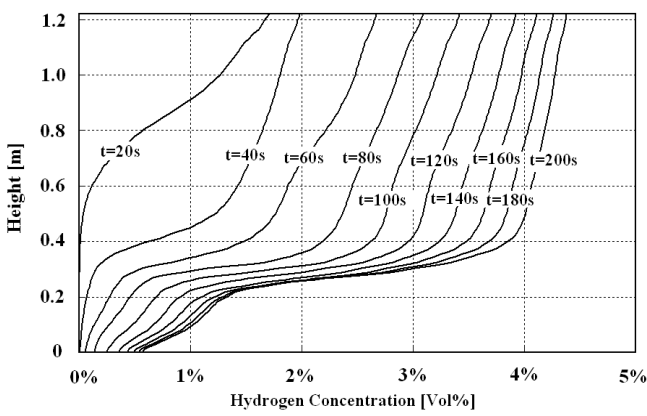

Figure 4: $\quad$-dir. distribution of hydrogen volumetric concentration at each time step.

Figure 4 shows y-directional distribution of hydrogen concentration every 20 seconds. After about 100 seconds from the start of leakage, the concentration in the lower part of the hallway converges within about $2 \%$. On the other hand, the concentration near the ceiling continues to increase, and the concentration stratification is formed. As is found by fig. 6 shown later, the circulation is formed in the hallway due to the transport of hydrogen from the inlet and the air taken from the door vent, and the iso-surface of concentration gradually descends from the ceiling almost in parallel to xz-plane.

Figure 5 shows the numerical results from Model 1 to Model 5 made to clarify the influence of ventilation position on concentration distribution. Here, only the steady state results are shown. Model 1 is the base model that is explained in fig. 1, and Models 2-4 are ones that changed only the position of the roof vent compared to Model 1 as in the lower left of fig. 5 . Model 5 is one that changed only the position of the door vent compared to Model 1 as in the lower right of fig. 5 . 

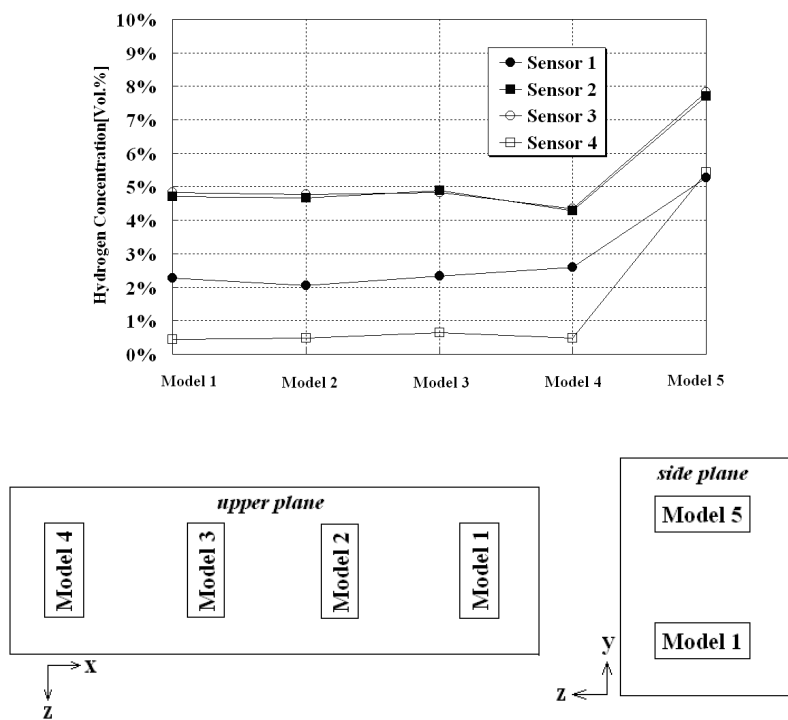

Figure 5: Variations of hydrogen concentration due to changes in vent position (upper) and the vent position in each model (lower).

In Model 4, the concentration at Sensor 2 and Sensor 3 becomes lower than that in Models 1-3. On the other hand, the concentration in Model 5 increases considerably at all sensor positions compared to those in all the other models. To explain this reason, the distribution of hydrogen concentration and velocity vectors are shown for Models 1 and 5 in fig. 6 . By changing the position of the door vent, air is taken into the space near the ceiling, and the hydrogen near the ceiling is transported to the lower part of the hallway. As a result, the concentration stratification mentioned before is highly disturbed and the concentration in the whole space increases. Therefore, the change in the position of the roof vent and the door vent has strong influence on the concentration distribution in the upper part and the lower part of the space, respectively.

In the next, changes in the concentration distribution in the hallway when the vent pressure varies due to external reasons are discussed. Figure 7 shows the changes in the hydrogen concentration when the pressure of the roof vent and the door vent is decreased in the range of 0.5-3.0 [Pa] from the prescribed value, separately. Although the hydrogen concentration in the lower part of the space does not change when the roof vent pressure is reduced, the concentration in the upper part decreases in the range of approximately $1-1.5 \%$. On the other hand, when the door vent pressure is reduced slightly, i.e., $0.5[\mathrm{~Pa}]$, the concentration increases considerably, and it becomes much higher than that when the door vent pressure is reduced by $3.0[\mathrm{~Pa}]$. To explain this reason, the effect of the door vent pressure on the spatial distribution of hydrogen concentration is shown in fig. 8 . When the door vent pressure is decreased by $0.5[\mathrm{~Pa}]$, hydrogen in the upper part of the space is transported to the lower part driven by air that shorts between the 
roof vent and the door vent. However, in this case the amount of hydrogen exhausted from the door vent is fewer than that in the case of the reduction by $3.0[\mathrm{~Pa}]$. Interpreting differently, slight pressure decrease reaches equilibrium when the exhausted hydrogen concentration is high because the dynamic pressure of hydrogen is very small.

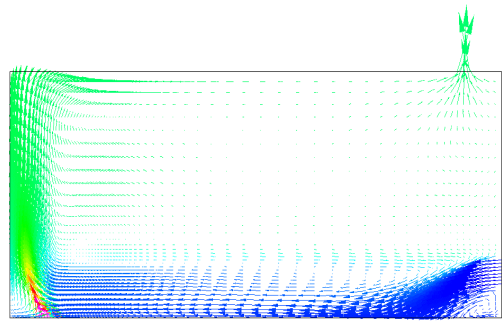

Model 1

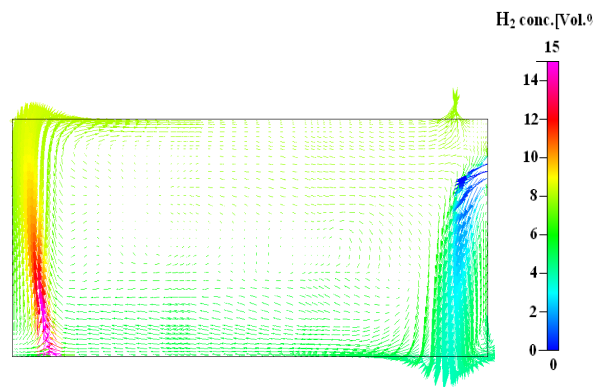

Model 5

Figure 6: The effect of vent position on hydrogen concentration.
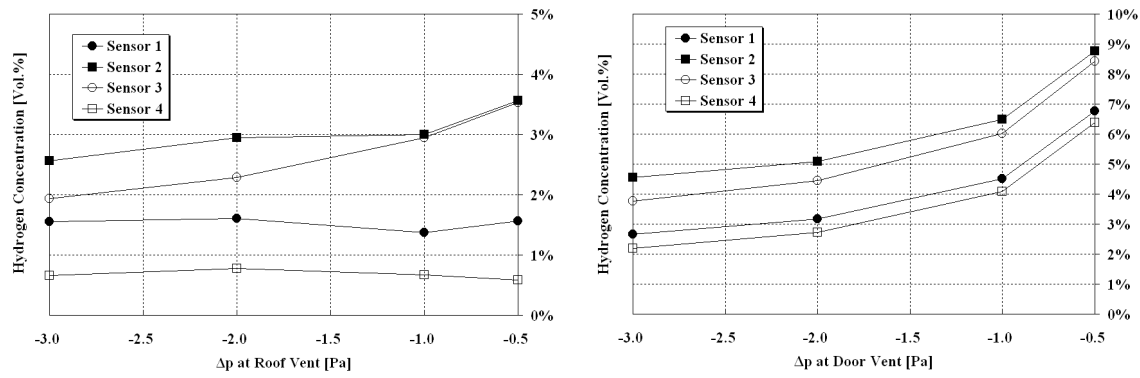

Figure 7: The effects of the roof vent pressure and door vent pressure on hydrogen concentration.

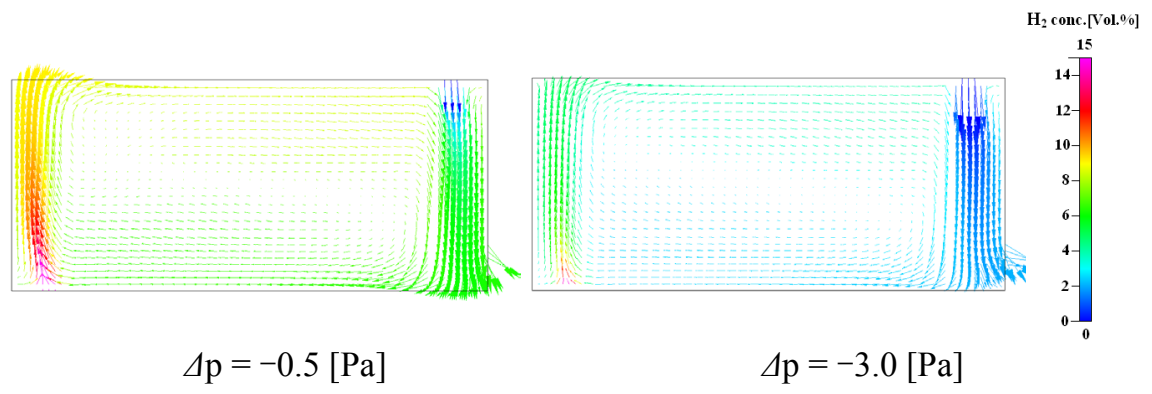

Figure 8: The effect of the door vent pressure on spatial hydrogen concentration. 

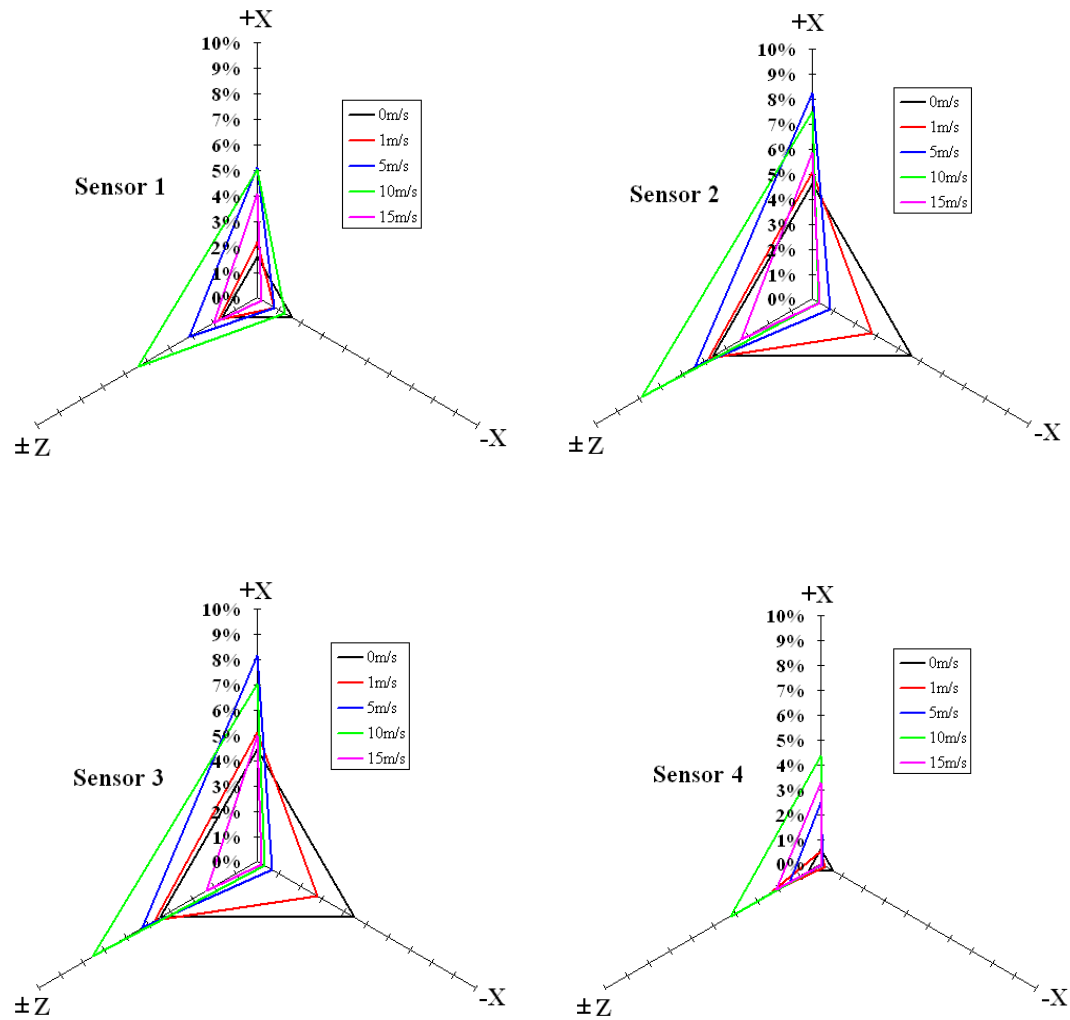

Figure 9: The effects of surrounding atmospheric currents on the distribution of hydrogen.

Based on the above discussion, in order to prevent the dispersion and accumulation of hydrogen, it is considered to be necessary to modify the shape of the space, or the vent positions so as not to disturb the concentration stratification in the space when leakage even if the pressure conditions change.

\subsection{The effect of atmospheric currents}

To show the effects of surrounding atmospheric currents on the distribution of hydrogen concentration, the direction and the magnitude of velocity are varied, i.e., $+\mathrm{x},-\mathrm{x}, \mathrm{z}$ direction, and $0,5,10$ and $15[\mathrm{~m} / \mathrm{s}]$, respectively. In this subsection, only the results of steady computation are shown. Figure 9 shows the variation of concentration at each sensor position. In the figure, each axis and the graduation correspond to the direction of atmospheric current, the volumetric hydrogen concentration, respectively. When the direction of atmospheric current is $-\mathrm{x}$, the concentration is decreased at all sensor positions compared to the case of calm atmosphere, i.e., the case of $U=0[\mathrm{~m} / \mathrm{s}]$ in the figure. On the other hand, when the direction of atmospheric current is $+\mathrm{x}$ and $\mathrm{z}$, the concentration increases considerably especially at the velocity magnitude of $10[\mathrm{~m} / \mathrm{s}]$. At these 
conditions, the increase of concentration in the lower part of the hallway is remarkable. These results mean that there are conditions that increase concentration in the hallway considerably according to the relationship with the outside environment. Therefore, when constructing a partially open space, or optimizing it computationally, it is necessary to take into consideration such relationship that augments hydrogen dispersion and accumulation.

\section{Conclusions}

Numerical Simulation of hydrogen dispersion in a partially open space is performed in this paper. The transient behavior of hydrogen and the process of hydrogen accumulation in the space are discussed. Also, the effects of changing vent positions, vent conditions and surrounding atmospheric currents on the distribution of hydrogen concentration are shown. Based on the results, conditions for preventing dispersion and accumulation of hydrogen in the space, and the importance of incorporating relationship with outside environment in computation, are discussed.

(1) Because of the transportation of hydrogen from the inlet orifice and air taken in from the door vent, the circulation is formed and the concentration stratification is maintained.

(2) The change in the position of the roof vent affects the concentration distribution only in the upper part of the space. By changing the position of the door vent toward the ceiling, transportation of hydrogen near the ceiling to the lower part of the space is promoted, and the concentration in the whole space increases considerably.

(3) The pressure reduction of the roof vent only decreases the concentration in the upper part of the space. On the other hand, the slight reduction in the pressure of the door vent, the concentration in the whole space increases considerably.

(4) In order to prevent the dispersion and accumulation of hydrogen, it is considered to be necessary to modify the shape of the space, or the vent position so as not to disturb the concentration stratification in the space when leakage even if the pressure conditions change.

(5) When constructing a partially open space, or optimizing it computationally, it is necessary to take into consideration such relationship with the outside environment that conditionally augments hydrogen dispersion and accumulation.

\section{Acknowledgement}

This research has been supported by the $21^{\text {st }}$ Century COE Program "Integration Technology of Mechanical Systems for Hydrogen Utilization" at Kyushu University. 


\section{References}

[1] Schefer, R.W., Houf, W.G., San Marchi, C., Chernicoff, W.P. \& Englom, L., Characterization of leaks from compressed hydrogen dispensing systems and related components. International Journal of Hydrogen Energy, 31, pp. 1247-1260, 2006.

[2] Venetsanos, A.G., Huld, T., Adams, P. \& Bartzis, J.G., Source, dispersion and combustion modelling of an accidental release of hydrogen in an urban environment. Journal of Hazardous Materials, A105, pp. 1-25, 2003.

[3] Schmidt, D., Krause, U. \& Schmidtchen, U., Numerical simulation of hydrogen gas releases between buildings. International Journal of Hydrogen Energy, 24, pp. 479-488, 1999.

[4] Olvera, H.A. \& Choudhuri, A.R., Numerical simulation of hydrogen dispersion in the vicinity of a cubic building in stable stratified atmospheres. International Journal of Hydrogen Energy, 31, pp. 2356-2369, 2006.

[5] Swain, M.R., Swain, M.N., Passive ventilation systems for the safe use of hydrogen. International Journal of Hydrogen Energy, 21(10), pp. 823-835, 1996.

[6] Swain, M.R., Grilliot, E.S. \& Swain, M.N., Risks incurred by hydrogen escaping from containers and conduits. Proc 1998 US DOE Hydrogen Prog Rev, NREL/CP-570-25315, 1998.

[7] Matsuura, K., Kanayama, H., Tsukikawa, H. \& Inoue, M., Researches on hydrogen dispersion behavior in a partially open space. Journal of Hydrogen Energy Systems Society of Japan, 31(2), pp. 50-57, 2006.

[8] Bird, R.B., Stewart, W.E. \& Lightfoot, E.N., Transport phenomena, $2^{\text {nd }}$ ed., Wiley: NY, 2002. 